\title{
New Concept of Bipolar Disorders
}

Ru-Band $\mathrm{Lu}^{1,2,3,4,6}$, Chia-Ling $\mathrm{Li}^{1}$ and Yi-Lun Chung ${ }^{1,5}$

${ }^{1}$ Department of Psychiatry, National Cheng Kung University Hospital, College of Medicine, National Cheng Kung University, Tainan, Taiwan

${ }^{2}$ Institute of Behavioral Medicine, National Cheng Kung University Hospital, College of Medicine, National Cheng Kung University, Tainan, Taiwan

${ }^{3}$ Institute of Allied Health Sciences, College of Medicine and Hospital, National Cheng Kung University, Tainan, Taiwan

${ }^{4}$ Addiction Research Center, National Cheng Kung University, Tainan, Taiwan

5 Institute of Basic Medical Sciences, National Cheng Kung University, Tainan, Taiwan

${ }^{6}$ Center for Neuropsychiatric Research, National Health Research Institutes, Miaoli, Taiwan

*Correspondence author: Ru-Band Lu, Department of Psychiatry, National Cheng Kung University Hospital, College of Medicine, National Cheng Kung University, Tainan, Taiwan, E-mail: rblu@mail.ncku.edu.tw

Received date: June 15, 2016; Accepted date: June 15, 2016; Published date: June 20, 2016

Copyright: ( 2016 Lu RB, et al. This is an open-access article distributed under the terms of the Creative Commons Attribution License, which permits unrestricted use, distribution, and reproduction in any medium, provided the original author and source are credited.

\section{Editorial}

Bipolar disorder (BP) characterized by a dysregulation of mood, impulsivity, risky behavior, and interpersonal problems, is a recurrent and often chronic psychiatric illness. BP is the sixth leading cause of disability-adjusted life years worldwide among persons aged 15 to 44 years [1]. It is associated with functional impairment elevated suicide rates, and utilization of mental health systems. The two most common and severe subtypes are bipolar I disorder (BP-I) and bipolar II disorder (BP-II). Although some researchers have questioned whether BP-II is simply a milder form of BP-I or is a distinct disorder, researchers have documented that BP-I and BP-II have different etiologies, including genetics, variation, and characteristics during the course of the illness [2]. Long-term follow-ups show that patients with BP-II have a more chronic course, more mood episodes, more major and minor depressive episodes, and shorter inter-episodes, which last longer than those of patients with BP-I [3]. The lifetime prevalence rate of BP-I is about $2.4 \%$ [4] while BP-II has been perceived as a common disorder with a prevalence of approximately $3-11 \%$ [5]. However, BP, and especially BP-II, is commonly under-recognized, even in psychiatric settings [6]. As many as more than $40 \%$ of patients with BP are initially misdiagnosed, and it may take years before those patients receive a correct diagnosis and appropriate treatment [7]. In our previous study, we found that the misdiagnosis rate of different subtypes of BP is much higher in Taiwan than in Western countries [8]. The current DSM-IV diagnostic criteria for BP-I and BP-II are the same except for different durations of hypomania and manic episodes: for BP-I it is more than 7 days and for BP-II more than 4 days [9]. This may also account for the difficulty in differentiating BP-I and BP-II and for the frequent misdiagnosis of BP-II. Even when correctly diagnosed, fewer than $50 \%$ patients are successfully treated [10], and $10-15 \%$ may eventually die as a result of suicide.

\section{Bipolar disorder as a neurodegenerative disease}

Evidences suggest that neuronal degeneration may relate to the etiology and progression of bipolar disorder. Imaging studies suggested that ongoing neuronal atrophy accompanies the disorders. PET images of the cerebral blood flow and the rate of glucose metabolism, both indicative of brain activity, detected a reduced activity in the subgenual prefrontal cortex during the bipolar depression. This decrement in activity in part corresponds to a reduction of cortical volume. Magnetic resonance imaging demonstrating the reduced mean volume on grey matters. In BP, abnormalities of the third ventricle, frontal lobe, cerebellum, and possibly the temporal lobe are also noted. These observations suggested a neuronal dysfunction and possibly neuronal loss may be involved in the pathogenesis of BP $[11,12]$. Therefore, BP might represent a neuro-degeneration disease. In addition, BP may be associated with induction of a lot of endotoxins and exotoxins which might increase neurotoxins as well as decrease of neurotrophic factors due to overactivate microglial cell and inhibit astroglia cell. Those effects may induce pro-inflammatory factors such as TNF- $\alpha$, C-reactive protein, interleukins, etc. and decrease BDNF etc. which will cause neuron damage or necrosis. The vicious cycle will lead to progressive worsening of the disease.

\section{Treatment for BP-II: Beyond mood stabilizer or antipsychotics}

While the pharmacological guidelines for treatment are well established [13], treatment for BP remains less than ideal, especially BP-II before 2000, not only BP-II has been misdiagnosed but scholars also wonder whether BP-II need treatment [5]. Most individuals still have breakthrough episodes or significant residual symptoms while on medication. In addition, functional deficits often remain even when patients are in remission. Moreover, most BP-II patients are newly diagnosed who have not taken any mood stabilizer or antipsychotics in the past. Because many patients with BP remain symptomatic even when fully adherent to their medication regimens, greater understanding of the pathogenesis of this illness and novel treatment modality other than current regimen of mood stabilizers and antipsychotics is needed. In our studies, we found that after adequate treatment, the BP-II had better functional improvement than the BP-I while excluding comorbidities, such as substance abuse [14].

\section{Novel treatment model: Memantine as an anti-inflammatory and neuroprotective agents}

Memantine used to be recognized as a noncompetitive N-methyl-Daspartate receptor antagonist. We found that memantine may inhibit brain inflammatory response through its action on neuroglial cells and provide neurotrophic effect. Mechanistic studies revealed that the high potency of small dosage of memantine is due to its dual actions: an anti-inflammatory effect by reducing the activity of microglia and an increase in the release of neurotrophic factors, such as BDNF, GDNF by astroglia [15] (For Hung-Ming Wu, Jau-Shyong Hong, Ru-Band Lu$^{*}$. US Patent 2009-0118376 A1. May 07, 2009). We found that even $1 / 100$ dosage of memantine $(0.2 \mathrm{mg} / \mathrm{kg})$ may be effective in opioid 
addictive behavior in rat by conditioned place preference [16]. Our clinical study was conducted that patients with VPA + memantine (5 $\mathrm{mg}$ /day) or VPA + placebo. Treating bipolar II with VPA + memantine may improve the plasma TNF- $\alpha$ level. However, adding-on memantine may not appear improve clinical symptoms. But the HDRS score changes were significantly associated with IL- 6 and IL- 1 level changes and changes in YMRS score changes with TNF- $\alpha$ level changes [17]. Thus, adding anti-inflammatory neuroprotective agents to the therapy might represent a better treatment paradigm for those patients.

\section{Dextromethorphan and Neuroprotective Effects}

Dextromethorphan (DM) has been used as an antitussive drug with little side effect for more than 50 years in clinics [18]. Liu et al. have reported that DM protects dopamine (DA) neurons against inflammation-mediated degeneration [19]. The mechanism of the neuroprotective effects of $\mathrm{DM}$ is associated with the inhibition of microglia activation but not with its N-methyl-D-aspartate (NMDA) receptor antagonist property. The evidence of benefits on auto-immune system with DM supports that DM as add-on therapy to valproic acid (VPA) might be more effective than VPA alone on the improvement of neuron degeneration in bipolar disorders. Our preliminary result suggested that DM might be effective in the treatment of BP patients. In addition, low plasma levels of DM $(5-100 \mathrm{ng} / \mathrm{ml})$ found in these patients suggested that this beneficial effect of DM is not mediated through the blockade of NMDA receptors. Instead, the antiinflammatory and neuroprotective actions may underlie the add-on therapeutic effect of DM to mood stabilizers in BP patients [20]. Our clinical studies have indicated that patients taking $30-60 \mathrm{mg} /$ day of DM showed a plasma DM concentrations of about $10-30 \mathrm{ng} / \mathrm{ml}(28-84$ $\mathrm{nM}$ ) which may not affect in NMDA receptor. The same low dose of DM causes a significant anti-inflammatory as well as a BDNFincreasing effect in bipolar disorder patients. (Use of Dextromethorphan in Treating Addictive Behavior or Bipolar Disorder. PATENT NUMBER: US 5,785,472 (07/22/2014). Patent for $\mathrm{Ru}-$ Band Lu.)

IL- 6 and IL- 1 level changes and changes in YMRS score changes with TNF- $\alpha$ level changes [16].

The anti-inflammation and neuroprotection may play one of important role of therapeutic perspective in $\mathrm{BP}$ and other major mental illness.

\section{References}

1. Murray CJ, Lopez AD (1996) Evidence-based health policy--lessons from the Global Burden of Disease Study. Science 274: 740-743.

2. Judd L, Akiskal HS, Schettler P, Coryell W, Maser J, et al. (2003). The comparative clinical phenotype and long term longuitudinal episode course of bipolar I and II: a clinical spectrum or distinct disorders. J Affect Disord 73: 19-32.

3. Judd LL, Akiskal HS (2003) Depressive episodes and symptoms dominate the longitudinal course of bipolar disorder. Curr Psychiatry Rep 5: 417-418.
4. Rihmer Z, Szadoczky E, Furedi J, Kiss K, Papp Z (2001) Anxiety disorders comorbidity in bipolar I, bipolar II and unipolar major depression: results from a population-based study in Hungary. J Affect Disord 67: 175-179.

5. Akiskal HS, Bourgeois ML, Angst J, Post R, Moller H, et al. (2000) Reevaluating the prevalence of and diagnostic composition within the broad clinical spectrum of bipolar disorders. J Affect Disord 59: 5-30.

6. Hirschfeld R, Calabrese JR, Weissman MM, Reed M, Davies MA, et al. (2003). Screening for bipolar disorder in the community. J Clin Psychiatry.

7. Calabrese JR, Hirschfeld RM, Reed M, Davies MA, Frye MA, et al. (2003) Impact of bipolar disorder on a U.S. community sample. J Clin Psychiatry 64: 425-432.

8. Merikangas KR, Herrell R, Swendsen J, Rossler W, Ajdacic-Gross V, et al. (2008) Specificity of bipolar spectrum conditions in the comorbidity of mood and substance use disorders: results from the Zurich cohort study. Arch Gen Psychiatry 65: 47-52.

9. American Psychiatric Association (2000) Diagnostic criteria from DSMIV-TR. Washington, DC.

10. Health, N.I.O.M (2002) Bipolar disorder.

11. Drevets WC (2000) Neuroimaging studies of mood disorders. Biol Psychiatry 48: 813-829.

12. Peng GS, Li G, Tzeng NS, Chen PS, Chuang DM, et al. (2005) Valproate pretreatment protects dopaminergic neurons from LPS-induced neurotoxicity in rat primary midbrain cultures: role of microglia. Brain Res Mol Brain Res 134: 162-169.

13. Suppes T, Dennehy EB, Hirschfeld RM, Altshuler LL, Bowden CL, et al. (2005) The Texas implementation of medication algorithms: update to the algorithms for treatment of bipolar I disorder. J Clin Psychiatry 66: 870-886.

14. Shan C, Lee SY, Chang YH, Wu JY, Chen SL, et al. (2011) Neuropsychological functions in Han Chinese patients in Taiwan with bipolar II disorder comorbid and not comorbid with alcohol abuse/alcohol dependence disorder. Prog Neuropsychopharmacol Biol Psychiatry 35: 131-136.

15. Wu HM, Tzeng NS, Qian L, Wei SJ, Hu X, et al. (2009). Novel neuroprotective mechanisms of memantine: increase in neurotrophic factor release from astroglia and anti-inflammation by preventing microglial activation. Neuropsychopharmacology: Official Publication of the American College of Neuropsychopharmacology 34: 2344-2357.

16. Chen SL, Tao PL, Chu CH, Chen SH, Wu HE, et al. (2012) Low-dose memantine attenuated morphine addictive behavior through its antiinflammation and neurotrophic effects in rats. J Neuroimmune Pharmacol 7: 444-453.

17. Lee SY, Chen SL, Chang YH, Chen PS, Huang SY, et al. (2014). The effects of add-on low-dose memantine on cytokine levels in bipolar II depression: a 12-week double-blind, randomized controlled trial.

18. Wang SC, Chou DT, Wallenstein MC (1977) Studies on the potency of various antitussive agents. Agents and Actions 7: 337-340.

19. Liu B, Hong JS (2003) Primary rat mesencephalic neuron-glia, neuronenriched, microglia-enriched, and astroglia-enriched cultures. Methods in Molecular Medicine 79: 387-395.

20. Chen SL, Lee SY, Chang YH, Chen PS, Lee IH, et al. (2014). Therapeutic effects of add-on low-dose dextromethorphan plus valproic acid in bipolar disorder. Eur Neuropsychopharmacol 24 :1753-1759. 